\title{
Pneumococcal Infection - Low Awareness as a Potential Barrier to Vaccination: Results of a European Survey
}

Hartmut Lode · Endre Ludwig · George Kassianos

To view enhanced content go to www.advancesintherapy.com

Received: March 14, 2013 / Published online: April 16, 2013

(c) The Author(s) 2013. This article is published with open access at Springerlink.com

\section{ABSTRACT}

Introduction: Elderly people and adults with chronic disease or compromised immune status are at increased risk of pneumococcal infection, with pneumonia being the most common serious presentation and a significant cause of morbidity and mortality. Most

\section{H. Lode}

Research Centre for Medical Studies, Institute of Clinical Pharmacology, Charité Universitätsmedizin Berlin, Reichsstr 2, Berlin, Germany

\section{E. Ludwig}

Division of Infectious Diseases, Department of Internal Medicine No. II, Semmelweis Medical University, Budapest, Hungary

G. Kassianos $(\square)$

Royal College of General Practitioners London Immunisation Lead, President British Global \& Travel Health Association, General Practitioner

The Ringmead Medical Centre, Bracknell, Berkshire, England, UK

Corresponding address: 61 Plough Lane, Wokingham, RG40 1RQ, UK

e-mail: gckassianos@btinternet.com

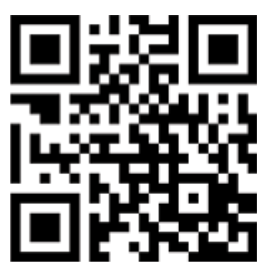

Enhanced content for Advances in Therapy articles is available on the journal web site: www.advancesintherapy.com
European countries have recommendations for pneumococcal vaccination but vaccination rates have remained low. In the present article, the authors present the results of a European survey that investigated the current level of awareness of pneumococcal infection among primary care physicians and specialists, and attitudes to vaccination in these physicians and members of the general public aged $>50$ years.

Methods: Primary care physicians $(n=1,300)$ and specialists $(n=926)$ from 13 Western European countries participated in online/face-to-face interviews, and a further 6,534 individuals aged $>50$ years from a population sample reflecting local socio-demographic structure participated in telephone/face-to-face interviews.

Results: Pneumonia was the most well-known of the pneumococcal infections amongst primary care physicians and specialists. However, there was a relatively low awareness of the term invasive pneumococcal disease (IPD), with only $50 \%$ of primary care physicians and $71 \%$ of specialists reporting knowledge of the term IPD. Key factors influencing a physician's decision to prescribe pneumococcal vaccination were the patient's health condition, recommendations from health authorities, and the tolerability of the vaccine. Perceptions regarding vaccination 
were good amongst the members of the general public; individuals did not fear vaccines or their side effects. The main drivers for vaccination were recommendations from a healthcare professional and, to a lesser extent, that vaccination provides reassurance against contracting a disease.

Conclusion: These findings highlight the low awareness of the term IPD in comparison with individual pneumococcal conditions. Given the importance of physician recommendations in encouraging patients to be vaccinated, primary care physicians need to be vigilant of patients at risk of pneumococcal infections in order to increase vaccination rates.

Keywords: Europe; Immunology; Infectious diseases; Invasive pneumococcal disease; Pneumococcal infection; Survey; Vaccination

\section{INTRODUCTION}

Infectious diseases in elderly people are a significant cause of morbidity and mortality in Europe in spite of the availability of antibiotics and vaccines for conditions such as pneumococcal infections, influenza, herpes zoster, and hepatitis A and B [1-5]. Multiple changes occur with age in both innate and adaptive immune systems, and this waning immunity (known as 'immunosenescence') as well as physiological changes associated with ageing make elderly individuals especially vulnerable to infectious diseases $[2,6]$. Pneumonia and influenza, in particular, tend to occur with greater severity in elderly individuals than in younger adults and are associated with poorer outcomes [4]. Adult populations at increased risk of pneumococcal infection are the elderly, those with chronic disease, such as lung or heart disease, diabetes, sickle cell anaemia, or asplenia, and those with compromised immune status, such as HIV-infected individuals [7].
The clinical spectrum of pneumococcal infections ranges from sinusitis and acute otitis media through to pneumonia and meningitis, with pneumonia being the most common serious presentation in adults $[5,7]$. Pneumococcal infections are classified as invasive pneumococcal disease (IPD) if Streptococcus pneumoniae is isolated from a normally sterile site such as the bloodstream or meninges [7]. Therefore, IPD is a microbiological rather than a clinical diagnosis, requiring specific testing usually within a secondary (i.e., hospital) setting. The term IPD is predominantly used by epidemiologists, vaccinologists, and paediatricians. Bacteraemic pneumonia is the most common IPD, comprising the overwhelming majority of adult cases [8-10].

S. pneumoniae is a leading cause of communityacquired pneumonia (CAP; i.e., pneumonia in individuals who have not been recently hospitalised) and thus most of the burden of serious pneumococcal infection in adults relates to pneumonia $[5,7]$. The incidence of CAP varies across both studies and countries; however, publications consistently report increasing incidence with increasing age $[5,11]$. Therefore, as the mean age of the population increases within Europe, it is expected that the burden of CAP is likely to increase. The burden of pneumococcal infections is also exacerbated by increasing numbers of individuals with chronic disease or HIV infection [12-15], and has remained high in adults aged $>50$ years despite the use of the 23 -valent purified capsular polysaccharide vaccine (PPV-23) in adults and the pneumococcal conjugate vaccine (PCV) in children [16].

The reported overall incidence of IPD in children and adults ranges from 10 to 23.2 per 100,000 in US and European studies conducted between 1995 and 2007, rising from 16.2 to 59.7 per 100,000 and 97 per 100,000 in adults aged $\geq 65$ and $\geq 90$ years, respectively [9, 10, 17-20]. 
Mortality associated with IPD increases with age in adults, reaching $10.6 \%$ in patients $\geq 65$ years of age and $20.6 \%$ in those aged $\geq 80$ years $[10,17]$. The following factors have been identified as being associated with fatal outcomes in patients with IPD: increasing comorbidity scores, neutropenia, HIV infection, chronic respiratory disease, and chronic liver disease [21].

In line with the significant burden of pneumococcal infections, recommendations for vaccinating elderly and vulnerable individuals against pneumococcal infections are available in most European countries. There is, however, considerable variability between countries concerning individuals regarded at risk of invasive pneumococcal infections, and these disparities appear to impact rates of pneumococcal vaccination across Europe. Higher rates of vaccine use have been reported in countries with age-based recommendations and public reimbursement (e.g., UK) than in those countries only recommending at-risk individuals (e.g., The Netherlands, Denmark, Portugal, and France) $[22,23]$. Lack of reimbursement may also explain low rates of vaccination in some countries (e.g., Austria, Finland, and Norway) with age-based recommendations [22].

The aim of this article is to describe the current level of awareness of pneumococcal infection among primary care physicians and specialists, as well as physicians' and the general publics' attitudes towards vaccination, based on findings from a European survey of physicians and individuals aged $>50$ years from the general public.

\section{MATERIALS AND METHODS}

\section{Survey}

A physician survey was conducted to determine pneumococcal disease awareness and attitudes to vaccination in 13 Western European countries (France, Germany, Italy, Spain, the UK, Austria, Greece, Portugal, Norway, Sweden, Switzerland, Finland, and Denmark). Primary care physicians (including internists in Germany) and specialists (pulmonologists, infectious disease specialists, other specialists: hygienists, geriatricians, internists, preventative medicine specialists) participated in 30-minute online or face-to-face (Greece and Portugal only) interviews held between November 292010 and January 242011.

Members of the general public aged $>50$ years also participated in a survey regarding their attitudes to vaccination. These individuals participated in 20-minute computer-assisted telephone or face-to-face interviews (in Germany and Spain) held between November 18 and December 292010.

This survey was conducted in accordance with the International Chamber of Commerce/ European Society for Opinion and Market Research (ICC/ESOMAR) code of practice, which defines ethical rules for professional market research companies and establishes safeguards for survey participants [24]. The survey methodology also complied with local laws and recommendations concerning data protection (e.g., in France, Article 29 of the Data Protection Act of 6 January 1978 amended in 2004 and the Commission nationale de l'informatique et des libertés [CNiL] recommendations [25]). Written informed consent was obtained from physicians participating via online interviews but not from physicians participating via face-to-face interviews or from members of the general public. Survey questions for both physicians and adults are available in the Supplementary Material (see Questionnaires 1 and 2).

This article does not contain findings from either interventional studies in humans or animal studies that have been performed by any of the authors. 


\section{Participant Eligibility and Recruitment}

Physicians were recruited from a European panel, which included 87,048 primary care doctors and 18,948 specialists (as defined above). Practicing physicians were included in the survey if they were aged between 30 and 65 years (inclusive), had been in practice for $\geq 3$ years, and spent $\geq 50 \%$ of their working time in direct patient care. Primary care physician and specialist selection was based on age and sex distribution according to country demographics and regional distribution. E-mail invites were sent to a sample of panellists, with e-mail and telephone follow-up to achieve the target number of participants. Survey respondents were paid for their participation.

Adults aged $>50$ years were recruited via random house sampling in Germany, and via interviewer selection, ensuring that quotas were fulfilled and that the individual was willing to participate, in Spain. Within the remaining countries, participants were recruited using the national phonebook information. The sample population was designed to reflect the socio-demographic structure of the national population available in each country. Adults aged $>50$ years who were representative of each country's demographic, in terms of age, sex, region, and socio-economic level, were selected for inclusion into the survey. In Germany, interviews were conducted faceto-face at the respondent's residence. In Spain, qualifying participants were invited for interview in the street and then came to a room for the interview. Members of the general public did not receive payment for their participation.

Both physicians and adults were excluded from the survey if they or someone in their family worked full-time in a pharmaceutical or market research company, or if they had taken part in a survey in the field of vaccination within the past 3 months.

\section{Statistical Analysis}

The survey findings were summarised descriptively with the percentage of primary care physicians, specialists, or individuals aged $>50$ years calculated for each response. Overall percentages (i.e., for all 13 countries) for each of these three groups were calculated, as well as percentages in each country. Mean frequency and severity scores (based on 7-point scales) for pneumococcal infections and some other common infectious diseases were also calculated for physicians. Statistical analysis of the levels of spontaneous awareness and probed awareness (i.e., physicians were presented with a list of terms) among the physicians were undertaken, using a two-sided t-test to compare awareness levels between the pneumococcal infection terms.

\section{RESULTS}

\section{Study Populations}

A total of 1,300 primary care physicians (100 from each country) participated in the survey from each of the 13 countries. The demographics of the primary care physicians varied by country. The percentage of male primary care physicians ranged from 44\% in Finland to 80\% in Denmark, and the percentage of physicians aged $>50$ years ranged from $19 \%$ in Spain to $72 \%$ in Portugal. The proportion of primary care physicians with $\geq 15$ years' experience also varied from $45 \%$ in Sweden to $81 \%$ in Italy.

The 926 specialists participating in the survey comprised a total of 86 specialists from Norway, Denmark, and Finland; 60 specialists each from Portugal, Austria, Switzerland, and Sweden; and 100 specialists each from the remaining six countries. These physicians were mostly respiratory specialists (ranging from 30\% to $92 \%$ depending on country) or infectious disease 
specialists (ranging from 5\% to 67\%). Other specialists included hygienists, geriatricians (or internists specializing in elderly medicine), and preventive medicine specialists within Italy, Germany, or Spain, respectively. The percentage of male specialists ranged from 63\% (Norway, Finland, and Denmark) to $88 \%$ (Germany). The proportion of specialists aged $>50$ years varied according to country (ranging from 16\% in the UK to $58 \%$ in Sweden), as did the proportion with $\geq 15$ years' experience (30\% in the UK and $73 \%$ in Italy).

A total of 6,534 adults aged $>50$ years participated in the survey (around 500 individuals from each of the 13 countries). Across these countries, the proportion of male individuals varied from $45 \%$ to $50 \%$. In Germany, $52 \%$ were aged $\geq 60$ years and, within the remaining 12 countries, between 31\% (Switzerland) and $60 \%$ (Portugal) were aged $\geq 65$ years. The proportion of retired individuals ranged from 29\% in Switzerland to 63\% in France.

\section{Disease Awareness Among Primary Care Physicians and Specialists}

Pneumonia was the most well-known of the pneumococcal infections. When physicians were asked to name pneumococcal infections, significantly more physicians named pneumonia than other pneumococcal infections $(P<0.001)$ : $78 \%$ of primary care physicians and $78 \%$ of specialists named pneumonia, 54\% and 60\% named meningitis, and 25\% and 36\% named sepsis/septicaemia, respectively. When presented with a list of pneumococcal infections, 93\% of primary care physicians and $96 \%$ of specialists stated that they knew about non-invasive pneumococcal pneumonia (Table 1), with awareness levels ranging from $83 \%$ to $99 \%$ and $91 \%$ to $100 \%$, respectively, across the 13 countries (data not shown). IPD, however, was the least well known term $(P<0.001$ for IPD vs. other terms); only $50 \%$ of primary care physicians (30-96\% within specific countries) and 71\% of specialists (56-98\%) knew about this term, compared with awareness levels of $75-94 \%$ and 92-97\%, respectively, for individual pneumococcal conditions categorised as IPD: meningitis, sepsis, bacteraemic pneumonia, and bacteraemia.

Physicians were also asked to grade the severity of various infectious diseases, including pneumococcal infections, using a 7-point scale, where 7 was extremely severe and 1 was not severe at all. Both primary care physicians and specialists rated seasonal influenza as less severe than any of the individual pneumococcal infections (meningitis, sepsis, IPD, and pneumonia; Fig. 1).

Table 1 Awareness of terms for pneumococcal infections among primary care physicians and specialists ${ }^{\mathrm{a}}$

\begin{tabular}{lll}
\hline & $\begin{array}{l}\text { Primary care physicians }(\%) \\
(n=1,300)\end{array}$ & $\begin{array}{l}\text { Specialists }(\%) \\
(n=926)\end{array}$ \\
\hline Pneumococcal pneumonia (non-invasive) & 93 & 96 \\
Pneumococcal meningitis & 94 & 96 \\
Pneumococcal sepsis & 91 & 97 \\
Bacteraemic pneumococcal pneumonia & 82 & 92 \\
Pneumococcal bacteraemia & 75 & 92 \\
Invasive pneumococcal diseases & $50^{*}$ & $71^{*}$ \\
\hline
\end{tabular}

${ }^{a}$ Physicians were presented with a list of terms and asked which of them they knew. ${ }^{*} P<0.001$ versus other terms 
The mean severity scores as rated by primary care physicians and specialists, respectively, varied from 5.7 to 6.6 and from 5.6 to 6.6 (based on the 7-point scale) for infections classified as IPD, as well as for the term IPD itself. Primary care physicians perceived pneumococcal sepsis and meningitis as very severe diseases (severity score: 6.6 for both) compared with a severity score of 4.9 for non-invasive pneumococcal pneumonia.

The frequency of pneumococcal infections as reported by specialists was higher than that reported by primary care physicians (mean frequency scores ranging from 2.8 to 4.7 and from 2.6 to 3.9 , respectively, on a 7-point scale,
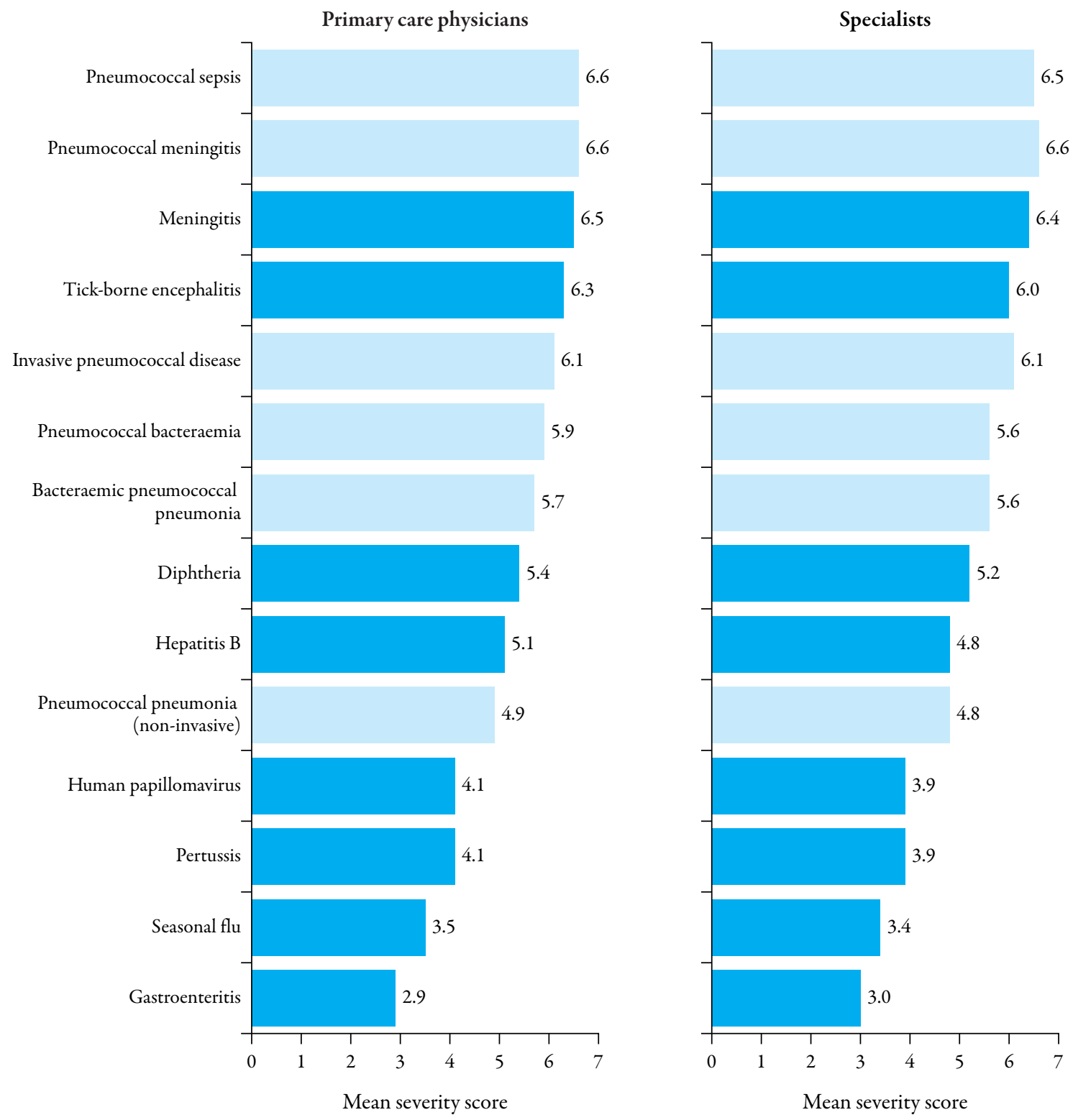

Fig. 1 Perceived severity of pneumococcal infections (light blue bars) compared with other infectious diseases (dark blue bars) among primary care physicians and specialists. Physicians (primary care physicians: $n=1,300$; specialists: $n=926$ ) were asked to grade the severity of the above infectious diseases using a 7-point scale, where 7 is extremely severe and 1 is not severe at all. The figure shows mean severity scores. The term meningitis refers to meningitis of unspecified aetiology 
where 7 was extremely frequent), and both groups of physicians perceived pneumococcal infections to be less frequent than other vaccinepreventable diseases such as gastroenteritis and influenza, which both had mean severity scores $>5$ (Fig. 2). The mean frequency scores for IPD (2.7 and 3.2), as rated by primary care physicians and specialists, respectively, were lower than those for non-invasive pneumococcal pneumonia (3.9 and 4.7) and bacteraemic pneumococcal pneumonia (3.3 and 3.9).

\section{Physicians' Attitudes to Vaccination}

Over $60 \%$ of primary care physicians and specialists selected the following as factors
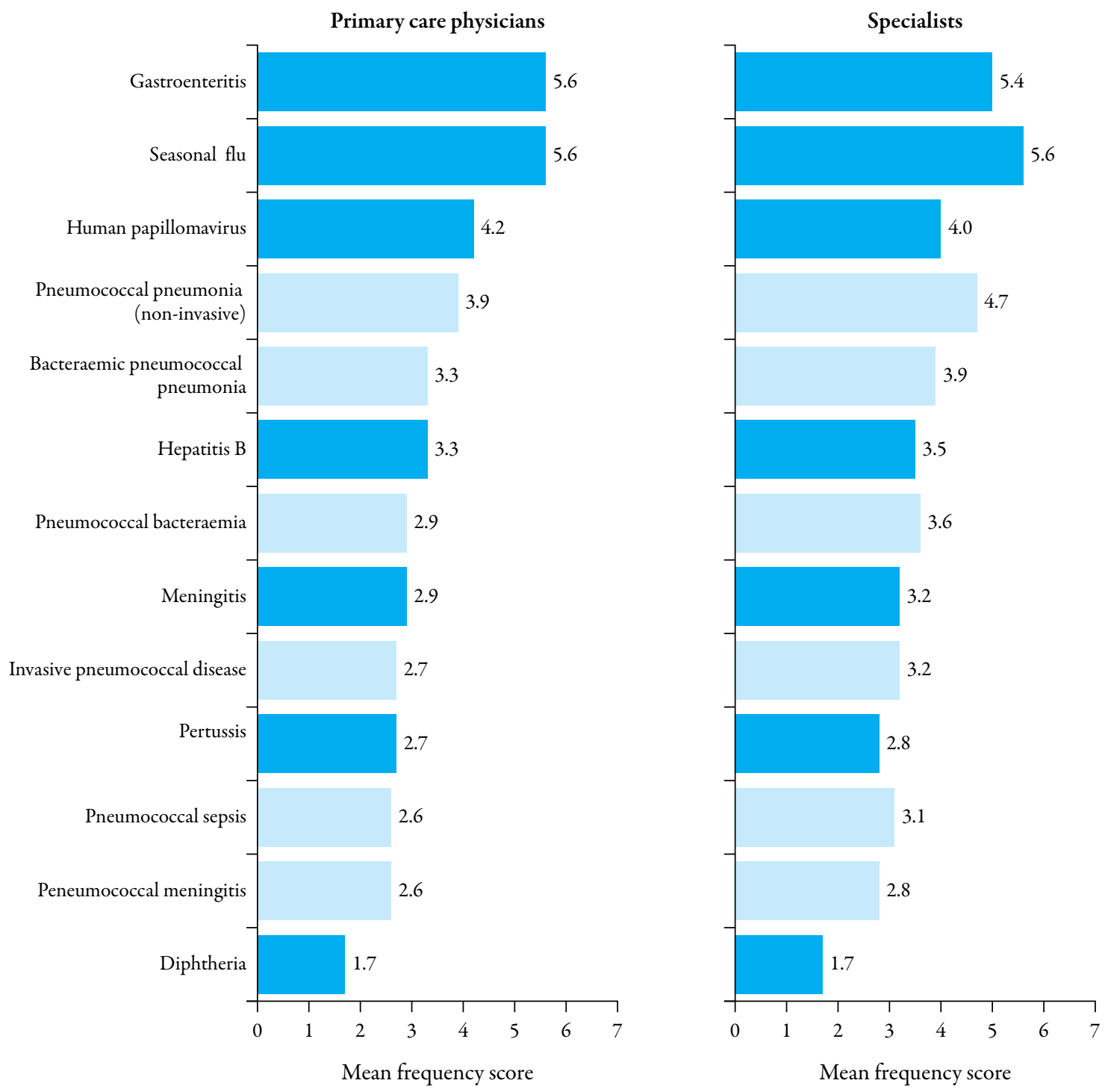

Fig. 2 Perceived frequency of pneumococcal infections (light blue bars) compared with other infectious diseases (dark blue bars) among primary care physicians and specialists. Physicians (primary care physicians: $n=1,300$; specialists: $n=926$ ) were asked to grade the frequency of the above infectious diseases using a 7-point scale, where 7 is extremely frequent and 1 is not frequent at all. The figure shows mean frequency scores. The term meningitis refers to meningitis of unspecified aetiology 
influencing their decisions regarding adult vaccination overall: patient's health condition, recommendations from health authorities, and tolerability of the vaccine (data not shown). For pneumococcal vaccination, the patient's health condition was also a key factor for recommending/prescribing vaccination (rated 6 or 7 on a 7 -point scale by $74 \%$ of primary care physicians and specialists), as were healthauthority recommendations, the patient's age, and tolerability of the vaccine (Fig. 3). When asked in what patient profile they would recommend and/or prescribe pneumococcal vaccination, most ( $\geq 76 \%)$ physicians selected at-risk or elderly individuals (Table 2, which includes physicians' definitions of these groups).
The main factors leading to a physician sometimes not recommending pneumococcal vaccination were that pneumococcal vaccination concerns only a subgroup of patients (selected by $67 \%$ and $77 \%$ of primary care physicians and specialists, respectively), the physician's mindset (i.e., vaccination is not at the top of the physician's mind), and lack of time (Table 2).

\section{General Public's Attitudes to Vaccination}

Of the adults aged $>50$ years included in the survey, $70 \%, 49 \%$, and $10 \%$ indicated that they had received tetanus/diphtheria, influenza, and/or pneumococcal vaccines, respectively, since the age of 18 years (see Supplementary Material - Table 1
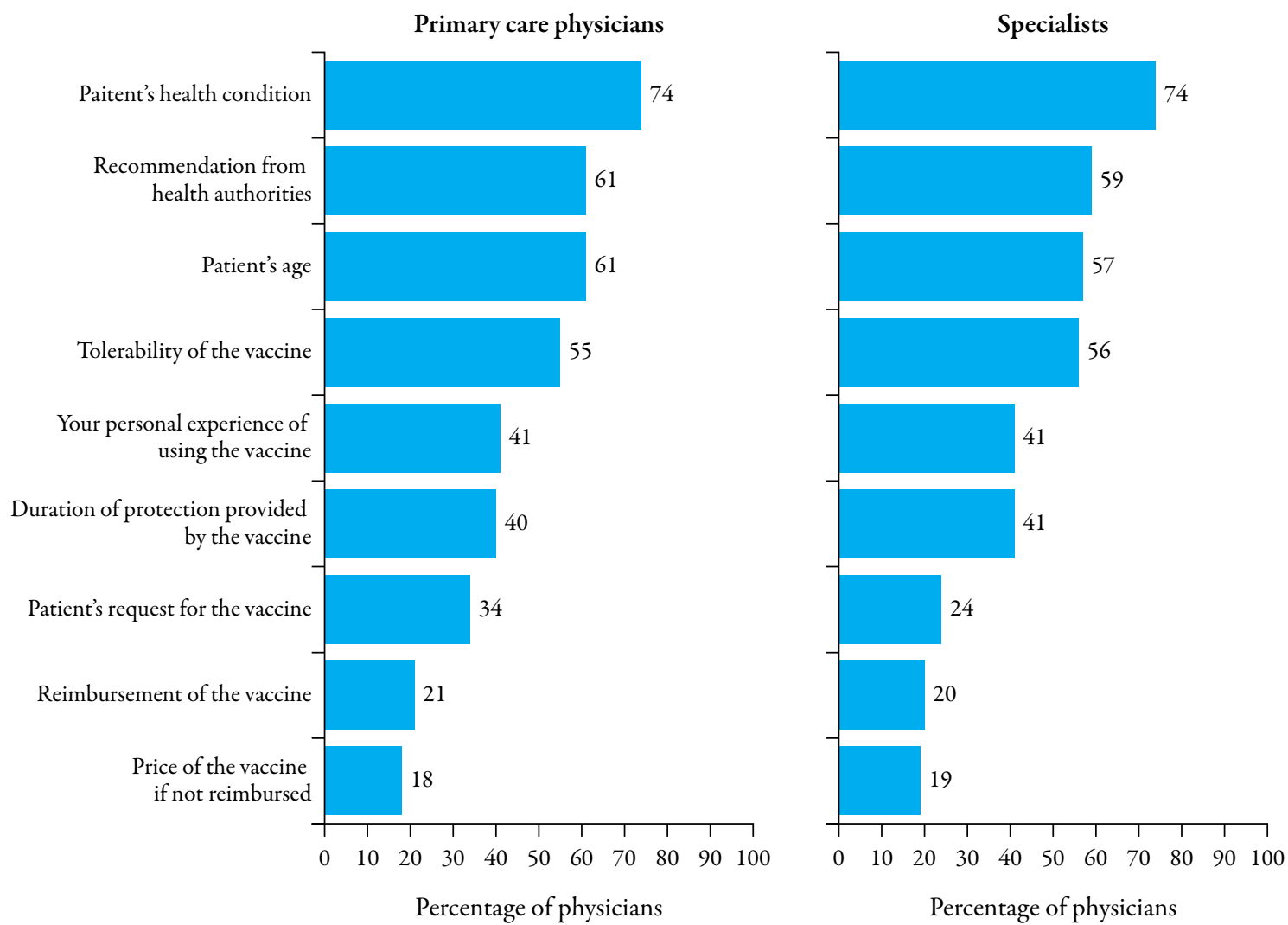

Fig. 3 Factors influencing physicians' decision making when recommending or prescribing pneumococcal vaccination for adult patients. Physicians (primary care physicians: $n=1,277$; specialists: $n=891$ ) were asked to indicate how important the above factors (using a 7-point scale) were when recommending or prescribing pneumococcal vaccination for adult patients. Figure shows percentage of physicians rating each factor 6 or 7 
for vaccination rates within each country). The percentage of individuals agreeing with statements concerning adult vaccination is shown in Table 3 . Over $60 \%$ of individuals in each country agreed that adult vaccines can save lives, while $\leq 35 \%$ considered vaccines to be unnecessary for treatable conditions and $\leq 25 \%$ were concerned about the side effects of vaccines. Key reasons for adults to be vaccinated were the need to protect against sickness (ranked as one of their top three reasons by $51-86 \%$ of individuals within each country), recommendations from a healthcare professional (18-65\%), and that vaccination provides reassurance against contracting a disease

Table 2 Factors affecting physicians' decision to prescribe pneumococcal vaccination in adults

\section{Primary care physicians (\%) Specialists (\%)}

$(n=1,267)$

$(n=918)$

Patient profile in which pneumococcal vaccination prescribed $^{a}$

At-risk patients ${ }^{\mathrm{b}}$

Elderly patients ${ }^{\mathrm{c}}$

Revaccination of patients

Patients who asked for the vaccination

People who care for elderly individuals

Healthcare professionals (to avoid infection from patients)

People who care for children

Other
93

76

44

43

22

19

12

7
96

76

43

36

24

23

16

8

Reasons why physicians may not recommend or prescribe pneumococcal vaccination ${ }^{\mathrm{d}}$

Vaccination concerns only specific groups of adults

67

77

Vaccination is not at top of mind

30

18

Lack of time

23

14

10

Not wanting to spend time convincing people not in favour of 17

vaccination

Not having time to discuss/explain benefits of vaccine

10

6

Doubts concerning the vaccine

6

10

Doubts concerning efficacy

4

8

Doubts concerning safety

\section{6} (6)

3

6




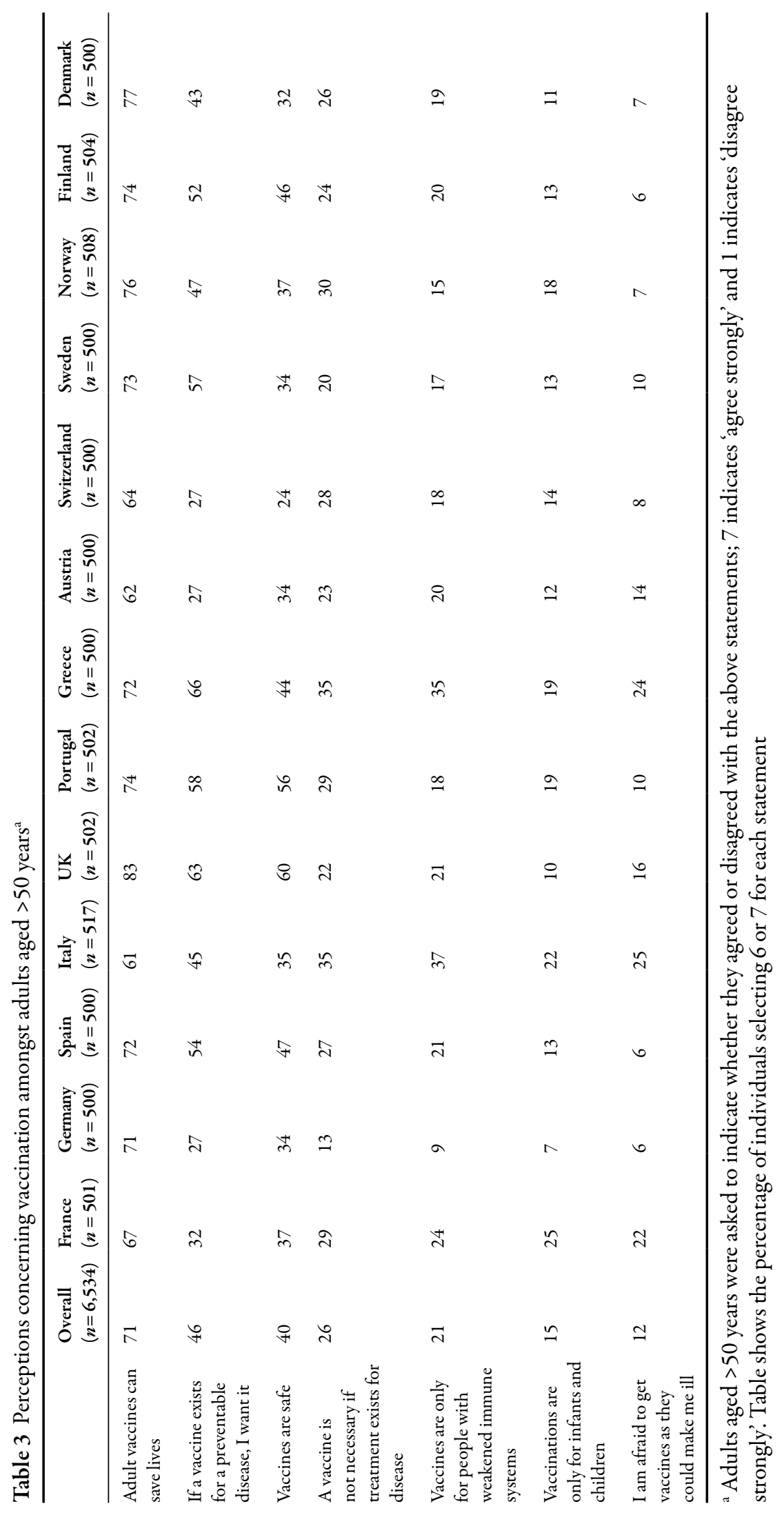




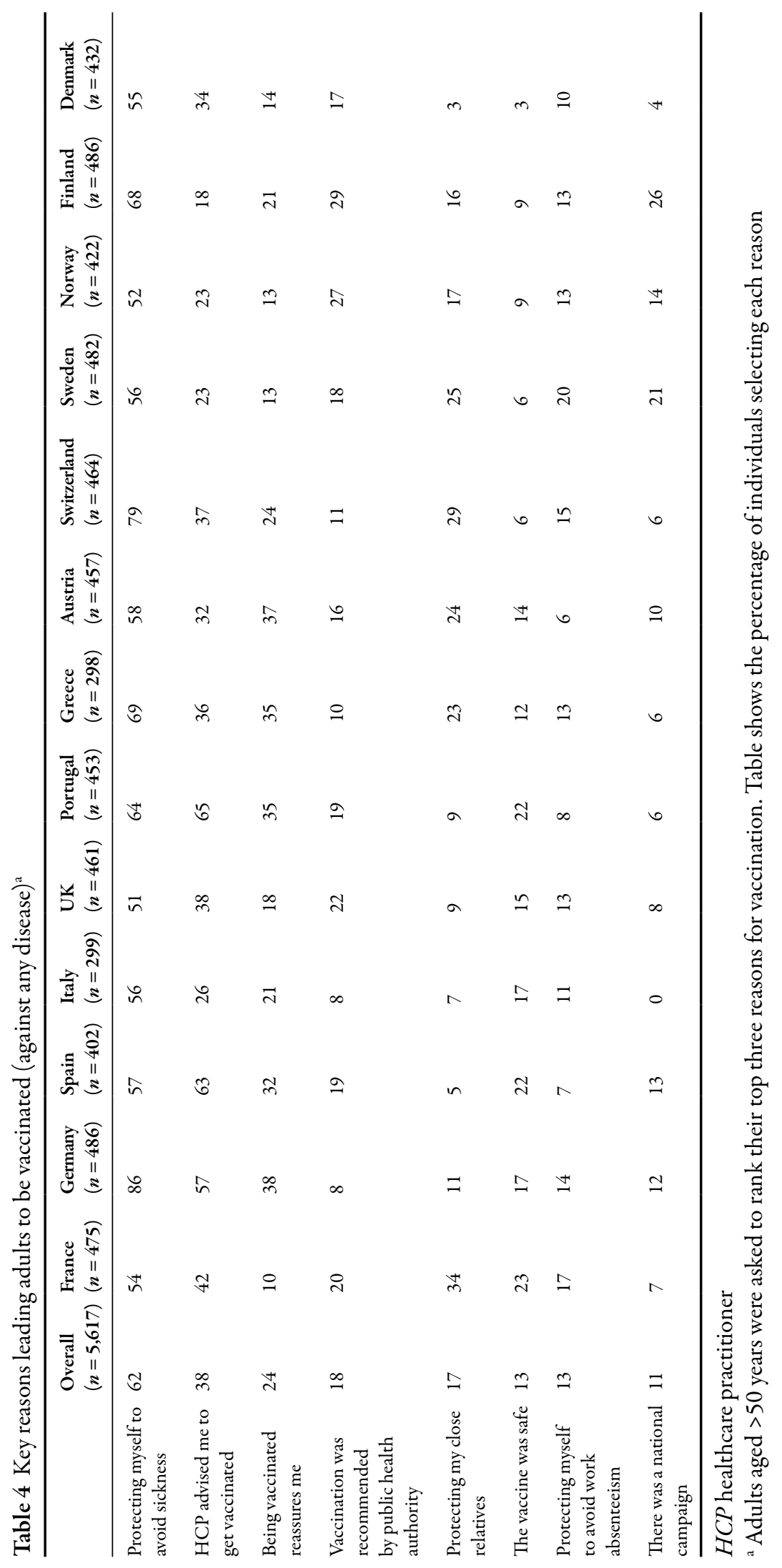




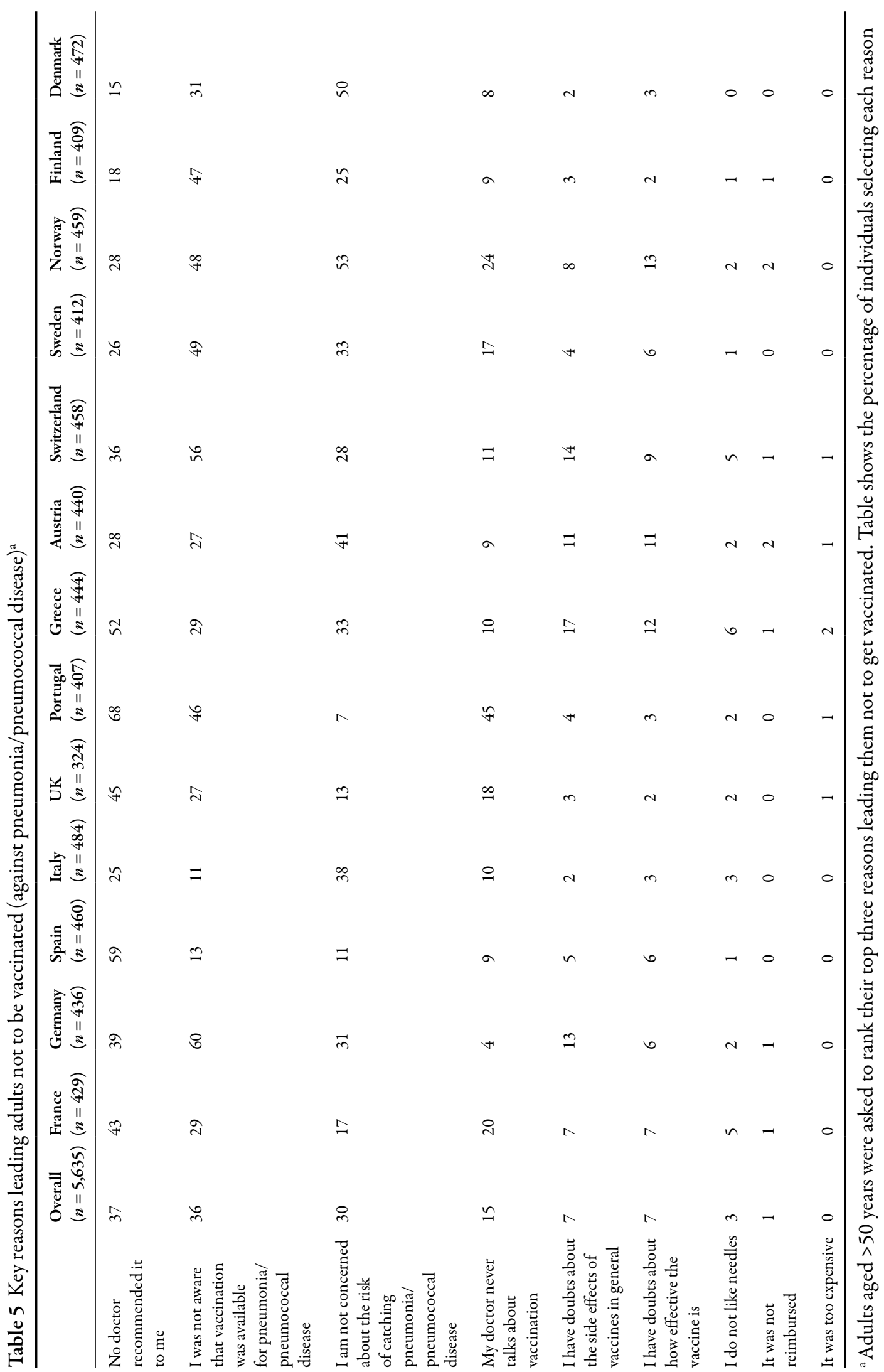


(10-38\%; Table 4). Similarly, individuals selected the following as key reasons for not receiving pneumococcal vaccines: lack of physician recommendation or vaccine awareness, and not being concerned about pneumonia/pneumococcal infections (Table 5).

\section{DISCUSSION}

\section{Disease Awareness}

Findings from this survey highlight the variability in knowledge/awareness levels of pneumococcal conditions amongst primary care physicians and specialists. Pneumonia was the most well-known of the pneumococcal infections, which is in line with the fact that pneumonia is the most common serious presentation of pneumococcal infections. The survey results, however, highlighted the relatively low knowledge of the term IPD. This may reflect the fact that IPD is not currently considered a relevant classification in everyday practice. This is perhaps not surprising given that IPD is not an 'independent' entity, except in rare cases of primary bacteraemia [26] and, also, that no significant differences have been identified in outcomes and mortality rates between CAP with or without pneumococcal bacteraemia [27].

Physicians within the survey considered pneumococcal infections to be severe. This is in line with morbidity and mortality data reported in the literature, especially in elderly individuals. For example, a Spanish study analysing hospital discharges and deaths related to pneumococcal pneumonia in adults aged >50 years between 2003 and 2007 calculated an annual hospitalisation rate of 1.09 cases per 1,000 , with a case-fatality rate of $11.6 \%$ for pneumococcal pneumonia [28]. Similarly, a 30 -day mortality rate of $9.6 \%$ was reported in a UK observational study conducted in adults hospitalised with pneumococcal non-invasive CAP between 2008 and 2010 [29].

The perceived frequency of pneumococcal infections tended to be lower in primary care physicians than in specialists, and both groups of physicians perceived pneumococcal infections to be less frequent than other vaccine-preventable diseases such as gastroenteritis and influenza. The perceived frequency of IPD was also lower than that of non-invasive pneumococcal pneumonia, as well as bacteraemic pneumococcal pneumonia, which is classified as IPD. These findings could be attributed to the fact that confirmation of IPD requires investigations, such as blood culture, which are not routinely undertaken in the community or on an outpatient basis. Therefore, it is a diagnosis that cannot easily be confirmed by primary care physicians. $S$. pneumoniae is a leading cause of lower respiratory tract infections (LRTIs), accounting for $38 \%$ of outpatient cases of CAP, $27 \%$ of inpatient cases of CAP, and $28 \%$ of intensive-care pneumonia cases, according to a recent review [5]. However, the exact contribution of pneumococcus to CAP is unknown, as a definite causative agent cannot be identified by conventional diagnostic methods (such as sputum culture) in most cases. A recent serological study conducted in hospitalised patients with CAP suggests that $S$. pneumoniae may actually account for $>50 \%$ of cases [30]. The incidence of pneumococcal infection in the community is unknown as most epidemiology studies have been conducted in hospitalised patients and primary care physicians will usually treat pneumonia without knowing the pathogen responsible.

\section{Factors Influencing Vaccination}

The survey also investigated physicians' attitudes to vaccination. Key factors influencing physicians to recommend or prescribe adult 
vaccines in general and pneumococcal vaccines in particular were an individual's condition and age, recommendations from health authorities, and tolerability of the vaccine. The main drivers that may lead a physician to sometimes not recommend pneumococcal vaccination were: pneumococcal vaccination concerns only a subgroup of patients, the physician's mindset, and lack of time. Doubts concerning the efficacy and safety of the vaccine and cost were low on the list of reasons against pneumococcal vaccination. This survey was conducted when PPV-23 was the only pneumococcal vaccine available. These findings, therefore, reflect the fact that PPV was reimbursed for patients considered to be at risk of pneumococcal infections in most countries included in this survey, and also suggest that doubts concerning PPV did not influence physician decisionmaking concerning pneumococcal vaccination.

Amongst the adults aged $>50$ years included in the survey, perceptions regarding vaccination were good: individuals considered vaccines to be lifesaving and they did not fear vaccines or their side effects. The main drivers leading adults to be vaccinated were the need to protect against sickness, recommendations from a healthcare professional, and (to a lesser extent) that vaccination provides reassurance against contracting a disease. The main barriers towards pneumococcal vaccination were lack of physician recommendation or vaccine awareness and not being concerned about pneumonia/ pneumococcal infections.

There was some variability between countries with respect to attitudes to vaccination from the adults aged $>50$ years, which may reflect variations in official vaccination recommendations and reimbursement between countries. However, a limitation of this survey was the differing methodologies employed across countries in terms of participant recruitment and interviews, and these differences may have introduced bias when comparing participant responses between countries.

\section{Importance of Vaccination Programmes}

Given the significant burden of pneumococcal infections, especially in elderly individuals, pneumococcal vaccination should be an important contribution to infectious disease prevention in an approach to lifelong health. As previously discussed, recommendations for vaccinating elderly and vulnerable individuals against pneumococcal infections vary from country to country, and this appears to impact coverage levels [22]. For example, the national pneumococcal immunisation programme within the UK includes all adults aged $\geq 65$ years and, according to an annual survey of English primary care practices, coverage of PPV within this age group was $68.3 \%$ in 2012 [23]. However, lower pneumococcal vaccination rates (20-30\%) have been reported in other European surveys in elderly individuals $[22,31,32]$. In the survey described here, $10 \%$ of adults aged $>50$ years indicated that they had received pneumococcal vaccines since the age of 18 years.

Findings from European surveys also suggest low pneumococcal vaccination rates amongst individuals with immunosuppression or chronic diseases [32-34]. Coverage rates in Italy, based on data collected from local health unit vaccination registers between 2000 and 2007, and cluster sampling among general practitioners, were around $20 \%$ in patients with chronic diseases [32]. Similarly, a German survey of immunosuppressed patients with chronic inflammatory diseases reported a pneumococcal vaccination rate of 9\% [34]. Within this survey, there was a marked discrepancy in terms of patients' willingness to be vaccinated and the actual provision of vaccination [34]. In a 
French study, a 3\% coverage rate was reported for HIV-infected individuals, with the most frequent reasons for non-vaccination reported as non-proposal by physicians, lack of expected effectiveness, and fear of immunovirological adverse effects [33].

Other surveys have suggested potential factors influencing pneumococcal vaccination, such as time spent with patients during 'well' visits, enhanced vaccination documentation, and patient factors (age, comorbidities, and quality of life) $[35,36]$. The general misconception that vaccination is an issue for early life/ childhood can be another barrier to adult vaccination. Patient-related barriers (e.g., fear of adverse events or injections) and healthcare professional-related issues (e.g., failure to endorse vaccination) have also been identified as factors influencing adult vaccination rates [37]. Low adult vaccination rates have led the European Union Geriatric Medicine Society and the International Association of Gerontology and Geriatrics to develop guidelines on vaccination of elderly individuals, with the aim of increasing adult vaccination coverage across Europe, thereby promoting healthy ageing by limiting the burden of vaccine-preventable infectious diseases [37].

Vaccination rates can be improved by nurseled programmes [38] or by administering pneumococcal vaccine when the patient attends the clinic for their annual influenza vaccination [39]. Computer systems, such as those used within the UK, that identify patients who have not been vaccinated and flag this information to the clinician within the clinical notes, may also help improve vaccination rates by allowing primary care physicians to promote pneumococcal vaccination at each patient visit. Pneumococcal infection awareness campaigns for the general public are a key initial step in improving vaccination rates amongst patients at risk [37].
PPVs have been available since 1983 and have been shown to reduce IPD in adults. However, data concerning the use of PPVs to prevent pneumococcal pneumonia or mortality are less clear [12, 40]. Specifically, there is limited evidence that PPV-23 is protective against pneumonia in elderly individuals or patients with chronic respiratory diseases, for whom the vaccine is recommended in many industrialised countries [40]. Over the past decade, a 7-valent PCV (PCV-7) has been available globally as part of a pneumococcal vaccination programme in children, and has resulted in a significant decrease in both IPD and mucosal disease in children [41-45] as well as also lower levels of antibiotic resistance [46]. There is evidence that elderly individuals have also, indirectly, benefited from this programme [47-50]. Higher-valent pneumococcal conjugate vaccines (PCV-10, PCV-13) have been available for use in infants and children in some countries since 2009 [44]. In Europe, PCV-13 is now available in some countries for the prevention of IPD in individuals aged $>50$ years [51], and for the prevention of IPD, pneumonia, and otitis media in infants and children from 6 weeks up to the age of 17 years [52]. PCV-10 is also available in Europe for immunisation against IPD and acute otitis media in infants and children from 6 weeks up to 5 years of age [53].

Immunological responses following PCV-13 vaccination, as measured by functional opsonophagocytic activity, have been demonstrated to be at least as good as those following PPV-23 for all serotypes, and significantly higher for 9/13 serotypes, in pneumococcal vaccine-naïve individuals aged 60-64 years $[54,55]$. PCV-13 re-vaccination, following either PCV-13 or PPV-23 vaccination, also results in renewed immunological response similar to that observed following the first PCV-13 dose. 


\section{CONCLUSION}

In summary, the survey findings reported here indicate a low awareness of the term IPD amongst primary care physicians. In contrast, there was greater awareness of pneumonia and of the individual pneumococcal conditions categorised as IPD, such as meningitis, sepsis, bacteraemic pneumonia, and bacteraemia. The survey findings also highlight the importance of physician recommendations for vaccination in encouraging patients to be vaccinated. There is, therefore, a need for primary care physicians to be more vigilant of patients at risk of pneumococcal infection in order to increase vaccination rates.

\section{ACKNOWLEDGMENTS}

This research was sponsored by Pfizer, Paris, France. The survey was administered by IPSOS Healthcare, Paris, France. The authors take full responsibility for the content of this article and thank Joanne Vaughan (Neostar Communications Ltd, Oxford, UK; funded by Pfizer, Paris, France) for her assistance in preparing the manuscript, including preparing the first draft in close collaboration with the authors and the collation of author comments. Pfizer also paid the article processing charges. The authors would also like to thank Olivia Bussod and Lídia Oliveira (Pfizer, France) for their comments and suggestions on the drafts of this manuscript and also Olivia Bussod for her involvement in the development of the survey. The authors did not receive honoraria in relation to the preparation of this manuscript. Dr. Kassianos is the guarantor for this article, and takes responsibility for the integrity of the work as a whole.

Conflict of interest. Hartmut Lode is a speaker and scientific adviser for Astellas, Bayer, OM Pharma, Novartis, Pfizer, Sanofi, and GSK.
Endre Ludwig has received honoraria for participating in advisory boards and giving lectures for Pfizer, Bayer, Astellas, MSD, and Novartis. George Kassianos has received honoraria for participating in advisory boards and/or giving lectures for AstraZeneca, Abbott, Bayer, Crucell/ Janssen, GSK, MSD, Pfizer, Sanofi Pasteur MSD, and Sigma-Tau.

Compliance with Ethics Guidelines. This survey was conducted in accordance with the International Chamber of Commerce/European Society for Opinion and Market Research (ICC/ ESOMAR) code of practice, which defines ethical rules for professional market research companies and establishes safeguards for survey participants. The survey methodology also complied with local laws and recommendations concerning data protection (e.g., in France, Article 29 of the Data Protection Act of 6 January 1978 amended in 2004 and the Commission nationale de l'informatique et des libertés [CNiL] recommendations). This article does not contain findings from either interventional studies in humans or animal studies that have been performed by any of the authors.

Open Access. This article is distributed under the terms of the Creative Commons Attribution Noncommercial License which permits any noncommercial use, distribution, and reproduction in any medium, provided the original author(s) and source are credited.

\section{REFERENCES}

1. Htwe TH, Mushtaq A, Robinson SB, Rosher RB, Khardori N. Infection in the elderly. Infect Dis Clin North Am. 2007;21:711-43, ix.

2. Liang SY, Mackowiak PA. Infections in the elderly. Clin Geriatr Med. 2007;23:441-56, viii.

3. Gusmano MK, Michel JP. Life course vaccination and healthy aging. Aging Clin Exp Res. 2009;21:258-63. 
4. Gavazzi G, Krause KH. Ageing and infection. Lancet Infect Dis. 2002;2:659-66.

5. Welte T, Torres A, Nathwani D. Clinical and economic burden of community-acquired pneumonia among adults in Europe. Thorax. 2012;67:71-9.

6. Weiskopf D, Weinberger B, Grubeck-Loebenstein B. The aging of the immune system. Transpl Int. 2009;22:1041-50.

7. Blasi F, Mantero M, Santus P, Tarsia P. Understanding the burden of pneumococcal disease in adults. Clin Microbiol Infect. 2012;18:7-14.

8. Sjostrom K, Spindler C, Ortqvist A, et al. Clonal and capsular types decide whether pneumococci will act as a primary or opportunistic pathogen. Clin Infect Dis. 2006;42:451-9.

9. Reinert RR, Haupts $S$, van der Linden $M$, et al. Invasive pneumococcal disease in adults in NorthRhine Westphalia, Germany, 2001-2003. Clin Microbiol Infect. 2005;11:985-91.

10. Robinson KA, Baughman W, Rothrock G, et al. Epidemiology of invasive Streptococcus pneumoniae infections in the United States, 1995-1998: opportunities for prevention in the conjugate vaccine era. JAMA. 2001;285:1729-35.

11. Fung HB, Monteagudo-Chu MO. Communityacquired pneumonia in the elderly. Am J Geriatr Pharmacother. 2010;8:47-62.

12. Moberley SA, Holden J, Tatham DP, Andrews RM. Vaccines for preventing pneumococcal infection in adults. Cochrane Database Syst Rev. 2008;CD000422.

13. Siemieniuk RA, Gregson DB, Gill MJ. The persisting burden of invasive pneumococcal disease in HIV patients: an observational cohort study. BMC Infect Dis. 2011;11:314.

14. Yin $\mathrm{Z}$, Rice $\mathrm{BD}$, Waight $\mathrm{P}$, et al. Invasive pneumococcal disease among HIV-positive individuals, 2000-2009. AIDS. 2012;26:87-94.

15. Grau I, Ardanuy C, Linares J, Podzamczer D, Schulze MH, Pallares R. Trends in mortality and antibiotic resistance among HIV-infected patients with invasive pneumococcal disease. HIV Med. 2009;10:488-95.

16. Elston JW, Santaniello-Newton A, Meigh JA, et al. Increasing incidence of invasive pneumococcal disease and pneumonia despite improved vaccination uptake: surveillance in Hull and East Yorkshire, UK, 2002-2009. Epidemiol Infect. 2012;140:1252-66.

17. Kyaw MH, Christie P, Clarke SC, et al. Invasive pneumococcal disease in Scotland, 1999-2001: use of record linkage to explore associations between patients and disease in relation to future vaccination policy. Clin Infect Dis. 2003;37:1283-91.

18. Trotter CL, Waight $\mathrm{P}$, Andrews $\mathrm{NJ}$, et al. Epidemiology of invasive pneumococcal disease in the pre-conjugate vaccine era: England and Wales, 1996-2006. J Infect. 2010;60:200-8.

19. Jansen AG, Rodenburg GD, de Greeff SC, et al. Invasive pneumococcal disease in the Netherlands: syndromes, outcome and potential vaccine benefits. Vaccine. 2009;27:2394-401.

20. Ardanuy C, Tubau F, Pallares R, et al. Epidemiology of invasive pneumococcal disease among adult patients in Barcelona before and after pediatric 7-valent pneumococcal conjugate vaccine introduction, 1997-2007. Clin Infect Dis. 2009;48:57-64.

21. Giner AM, Kuster SP, Zbinden R, Ruef C, Ledergerber $\mathrm{B}$, Weber R. Initial management of and outcome in patients with pneumococcal bacteraemia: a retrospective study at a Swiss university hospital, 2003-2009. Infection. 2011;39:519-26.

22. Fedson DS, Nicolas-Spony L, Klemets $\mathrm{P}$, et al. Pneumococcal polysaccharide vaccination for adults: new perspectives for Europe. Expert Rev Vaccines. 2011;10:1143-67.

23. Department of Health. Pneumococcal polysaccharide vaccine (PPV) uptake summary report. Available at: http://immunisation.dh.gov. uk/ppv-uptake-report-2012. Accessed Apr 92013.

24. World ESOMAR Research, ICC. ICC/ISOMAR International Code on market and social research. ESOMAR 2007. Available at: http://www.esomar. org/uploads/public/knowledge-and-standards/ codes-and-guidelines/ESOMAR_ICC-ESOMAR_ Code_English.pdf. Accessed Mar 272013.

25. CNIL. Decree No 2005-1309 of 20 October 2005 enacted for the application of Act No 78-17 of 6 January 1978 on Data Processing, Files and Individual Liberties (Amended by Decree 2007-451 of 25 March 2007) Consolidated on the 25th of March 2007. CNIL 2007. Available at: http://www. cnil.fr/fileadmin/documents/en/Decree\%2020051309.pdf. Accessed Mar 272013. 
26. Musher DM. Pneumococcal infections. In: Kasper DL, Fauci AS, eds. Harrison's Infectious Diseases. McGraw Hill Medical; 2010:374-85.

27. Bordon J, Peyrani P, Brock GN, et al. The presence of pneumococcal bacteraemia does not influence clinical outcomes in patients with community-acquired pneumonia: results from the Community-Acquired Pneumonia Organization (CAPO) International Cohort study. Chest. 2008;133:618-24.

28. Gil-Prieto R, Garcia-Garcia L, Alvaro-Meca A, Mendez C, Garcia A, de Miguel AG. The burden of hospitalisations for community-acquired pneumonia (CAP) and pneumococcal pneumonia in adults in Spain (2003-2007). Vaccine. 2011;29:412-6.

29. Bewick T, Sheppard C, Greenwood S, et al. Serotype prevalence in adults hospitalised with pneumococcal non-invasive community-acquired pneumonia. Thorax. 2012;67:540-5.

30. van Mens SP, Meijvis SC, Endeman $H$, et al. Longitudinal analysis of pneumococcal antibodies during community-acquired pneumonia reveals a much higher involvement of Streptococcus pneumoniae than estimated by conventional methods alone. Clin Vaccine Immunol. 2011;18:796-801.

31. Gavazzi G, Wazieres B, Lejeune B, Rothan-Tondeur $M$. Influenza and pneumococcal vaccine coverages in geriatric health care settings in France. Gerontology. 2007;53:382-7.

32. Martinelli D, Tafuri S, Caputi G, et al. Eight years of active proposal of pneumococcal 23-valent polysaccharide vaccine: survey on coverage rate among elderly and chronic patients. Am J Infect Control. 2010;38:e8-e15.

33. Mohseni-Zadeh M, Rey D, Batard ML, et al. Inadequate vaccination coverage in a French cohort of HIV positive patients [In French]. Med Mal Infect. 2010;40:683-90.

34. Teich N, Klugmann $\mathrm{T}$, Tiedemann A, et al. Vaccination coverage in immunosuppressed patients: results of a regional health services research study. Dtsch Arztebl Int. 2011;108:105-11.

35. Zimmerman RK, Nowalk MP, Tabbarah M, Hart JA, Fox DE, Raymund M. Understanding adult vaccination in urban, lower-socioeconomic settings: influence of physician and prevention systems. Ann Fam Med. 2009;7:534-41.
36. Krueger P, St Amant O, Loeb M. Predictors of pneumococcal vaccination among older adults with pneumonia: findings from the Community Acquired Pneumonia Impact Study. BMC Geriatr. 2010;10:44.

37. Michel JP. Updated vaccine guidelines for aging and aged citizens of Europe. Expert Rev Vaccines. 2010;9:7-10.

38. Lau D, Hu J, Majumdar SR, Storie DA, Rees SE, Johnson JA. Interventions to improve influenza and pneumococcal vaccination rates among community-dwelling adults: a systematic review and meta-analysis. Ann Fam Med. 2012;10:538-46.

39. Badertscher N, Morell S, Rosemann T, Tandjung R. General practitioners' experiences, attitudes, and opinions regarding the pneumococcal vaccination for adults: a qualitative study. Int J Gen Med. 2012;5:967-74.

40. Huss A, Scott P, Stuck AE, Trotter C, Egger M. Efficacy of pneumococcal vaccination in adults: a meta-analysis. CMAJ. 2009;180:48-58.

41. Foster D, Walker AS, Paul J, et al. Reduction in invasive pneumococcal disease following implementation of the conjugate vaccine in the Oxfordshire region, England. J Med Microbiol. 2011;60:91-7.

42. Pilishvili T, Lexau C, Farley MM, et al. Sustained reductions in invasive pneumococcal disease in the era of conjugate vaccine. J Infect Dis. 2010;201:32-41.

43. Simonsen L, Taylor RJ, Young-Xu Y, Haber M, May L, Klugman KP. Impact of pneumococcal conjugate vaccination of infants on pneumonia and influenza hospitalization and mortality in all age groups in the United States. MBio. 2011;2:e0309-10.

44. Reinert RR, Paradiso P, Fritzell B. Advances in pneumococcal vaccines: the 13-valent pneumococcal conjugate vaccine received market authorization in Europe. Expert Rev Vaccines. 2010;9:229-36.

45. Zhou F, Shefer A, Kong Y, Nuorti JP. Trends in acute otitis media-related health care utilization by privately insured young children in the United States, 1997-2004. Pediatrics. 2008;121:253-60.

46. Cohen R, Levy C, de La Rocque F, et al. Impact of pneumococcal conjugate vaccine and of reduction of antibiotic use on nasopharyngeal carriage of nonsusceptible pneumococci in children with acute otitis media. Pediatr Infect Dis J. 2006;25:1001-7. 
47. Whitney CG, Farley MM, Hadler J, et al. Decline in invasive pneumococcal disease after the introduction of protein-polysaccharide conjugate vaccine. N Engl J Med. 2003;348:1737-46.

48. Lexau CA, Lynfield R, Danila R, et al. Changing epidemiology of invasive pneumococcal disease among older adults in the era of pediatric pneumococcal conjugate vaccine. JAMA. 2005;294:2043-51.

49. Miller E, Andrews NJ, Waight PA, Slack MP, George RC. Herd immunity and serotype replacement 4 years after seven-valent pneumococcal conjugate vaccination in England and Wales: an observational cohort study. Lancet Infect Dis. 2011;11:760-8.

50. Myint TT, Madhava H, Balmer $P$, et al. The impact of 7-valent pneumococcal conjugate vaccine on invasive pneumococcal disease: a literature review. Adv Ther. 2013;30:127-51.

51. Pfizer Limited. Prevenar 13 suspension for injection summary of product characteristics. eMC. Available at: http://www.medicines.org.uk/ emc/medicine/22689/SPC/Prevenar+13+suspensio n+for+injection/. Accessed Feb 42013.
52. European Medicines Agency. Prevenar 13. European Medicines Agency 2013. Available at: http://www. ema.europa.eu/ema/index.jsp?curl=pages/medicines/ human/medicines/001104/smops/Positive/human_ smop_000452.jsp\&mid=WC0b01ac058001d127. Accessed Mar 42013.

53. GlaxoSmithKline Biologicals s.a. Synoflorix. Summary of product characteristics. European Medicines Agency 2013. Available at: http://www. ema.europa.eu/docs/en_GB/document_library/ EPAR_-_Product_Information/human/000973/ WC500054346.pdf. Accessed Mar 42013.

54. Jackson LA, Gurtman A, van Cleeff M. Immunogenicity and safety of a 13-valent pneumococcal conjugate caccine in pneumococcal vaccine naive adults, 50-64 years of age [abstract O426]. Clin Microbiol Infect. 2011;17:S85.

55. Jackson LA, Gurtman A, van Cleeff M. 13-valent pneumococcal conjugate vaccine (PCV13) enhances the response to subsequent PCV13 and 23-valent pneumococcal polysaccharide (PPSV23) vaccinations in adults 50 years and older [abstract LB-3]. Presented at the 49th Annual Meeting of the Infectious Diseases Society of America; October 2023, 2011; Boston, MA. 